Voix et Images

\title{
L'Histoire des idées au Québec. De Lionel Groulx à Paul-Émile Borduas
}

\section{Georges Vincenthier}

Volume 2, numéro 1, septembre 1976

Fernand Leduc

URI : https://id.erudit.org/iderudit/200019ar

DOI : https://doi.org/10.7202/200019ar

Aller au sommaire du numéro

Éditeur(s)

Les Presses de l'Université du Québec

ISSN

0318-9201 (imprimé)

1705-933X (numérique)

Découvrir la revue

Citer cet article

Vincenthier, G. (1976). L'Histoire des idées au Québec. De Lionel Groulx à

Paul-Émile Borduas. Voix et Images, 2(1), 28-46.

https://doi.org/10.7202/200019ar d'utilisation que vous pouvez consulter en ligne.

https://apropos.erudit.org/fr/usagers/politique-dutilisation/ 


\section{L'Histoire des idées au Québec De Lionel Groulx à Paul-Émile Borduas}

\section{L'ACTION NATIONALE : UNE RENAISSANCE DE LA PENSÉE QUÉBÉCOISE}

Après s'être enlisée dans les chemins marécageux d'une orthodoxie oiseuse et stérile, la pensée québécoise, stimulée par des penseurs comme $E$. de Nevers et $E$. Bouchette, aiguillonnée par l'action débordante d'Henri Bourassa, allait s'acheminer sur une voie plus créatrice. Lorsqu'en 1917, le premier numéro de l'Action française ${ }^{1}$ paraît c'est une ère nouvelle qui commence. Devant les piètres résultats obtenus jusqu'ici par la société québécoise, les rédacteurs de l'Action s'efforcent d'aller un peu plus de l'avant. Avec eux une rigoureuse dialectique de la survie nationale va s'engager. Pris dans un monde anglophone qui refuse de reconnaître le fait français, les jeunes intellectuels des années 20, aidés de quelques aînés, vont travailler à la revalorisation du phénomène linguistique et national: "Au sein d'une civilisation matérialiste et contre des politiciens souvent sans pudeur, l'école nationaliste fut à peu près seule à dresser une pensée ${ }^{2}$, dira d'eux un homme qui n'avait aucune raison de louanger la revue nationaliste, Pierre E. Trudeau. De fait l'équipe de l'Action française va réunir autour d'elle tous les intellectuels, tous les hommes d'affaires, tous les universitaires dans une action concertée pour unifier et clarifier la notion de nation canadienne-française, les objectifs qu'elle se propose et les moyens à mettre en œuvre pour les réaliser. Ce fut une grande entreprise de réunification et de réflexion intense. Pour cela et afin de donner à leur action une valeur plus grande et des chances de réussite plus concrètes, les dirigeants de la revue commencèrent une série d'enquêtes approfondies sur les différents problèmes de la société québécoise, ainsi que sur les groupes sociaux constituants. La jeunesse eut droit à son enquête, on détermina le rôle de la femme, les ouvriers eux-mêmes eurent une fonction déterminée. Les plus connues et les plus intéressantes de ces enquêtes furent "Notre avenir politique» ainsi que "l'Avenir de notre bourgeoisie".

Avant de passer à ces analyses. spécialisées, il fallait cependant se donner une ligne de conduite et «pour mettre l'ordre dans les choses, en mettre d'abord dans les esprits ${ }^{3}$ ". Cet ordre à mettre dans les esprits fut la principale préoccupation de la revue. Dès le premier numéro, É. Mont- 
petit, dans un article intitulé "Vers la supériorité", éclairait les lecteurs sur les projets des nouveaux intellectuels. Ne se fier qu'au titre de l'article serait trompeur. On pourrait revoir là une manifestation de la grandiloquence de la pensée orthodoxe. Il y a bien sûr un relent de cette pensée. Mais nous retiendrons surtout ceci : cette supériorité ne pourra être atteinte que par le travail. Elle n'est plus innée, comme l'avait laissé croire AdolpheBasile Routhier ou F.-L. Laflèche. Car présentement, pour Montpetit, non seulement le peuple québécois n'est pas supérieur, mais il est de beaucoup inférieur à ses voisins. Et alors, il reprend la campagne de Bouchette et prêche que la «conquête économique doit être pour nous la réalité de demain ". Cette conquête économique, pour Montpetit comme pour tout le mouvement dans lequel il s'inscrit, ne peut être un souci bassement individualiste. Nous touchons là une caractéristique fondamentale de l'Action française: l'effort de l'individu n'a de sens et de succès qu'en autant qu'il s'inscrit dans un mouvement collectif où l'individu supporté par un idéal national redonne en retour à cet idéal toutes les énergies nécessaires à la montée du peuple et à la réalisation du projet collectif:

L'individu n'est fort que par le groupe qui l'utilise et le complète. II serait exagéré de lui demander de tout savoir, de tout prévoir. II suffit 'que, instruit de son rôle, exécutant l'acte qui lui est confié, il assure l'ceuvre que poursuit la collectivité 4 .

Voilà en quelques lignes le sens du nationalisme de l'Action française. C'est en faisant un voyage aux États-Unis en 1918 que Montpetit complètera définitivement sa pensée économique nationale. En visitant l'encore jeune Université Berkeley et voyant tout ce que la richesse matérielle peut donner à l'esprit et à l'idéal, il reviendra avec quelques notes réalistes qui le rapprochent de Parent et de Bouchette. Mais qui se souvenait de ces penseurs ? Aussi les constatations de Montpetit estomaquèrent-elles. Sur le budget de trois millions de l'Université Berkeley, il fera un commentaire dont Louis-A. Paquet et H.-R. Casgrain eussent pâli d'épouvante:

Et si, comme nous disons avec une moitié de raison, «l'argent n'est pas tout», on conviendra que c'est déjà quelque chose ${ }^{5}$.

Si modérée que soit cette affirmation, elle proposait à la pensée québécoise un bon bain dans la réalité. Aux États-Unis, loin de voir le bas matérialisme, le jeune professeur d'économique constate que:

Partout s'affirme une préoccupation d'art et de beauté, une volonté d'enseigner non plus seulement les riches, mais les petits, les humbles, les foules. Partout s'élèvent des écoles, des collèges, des universités, des bibliothèques, des musées ${ }^{6}$.

De ses réflexions, de ses observations, É. Montpetit tire une pensée tout entière préoccupée du progrès social et économique de la nation canadienne-française. Mais lui et toute la société qui l'entoure sont encore trop près de l'idéalisme du $X X^{\ominus}$ siècle. Même avec les meilleures intentions, ils ne réussirent jamais à incarner dans des projets concrets leur idéal de conquête économique. Parler d'économie dans une chaire universitaire, écrire à ce sujet de brillants articles ne changent que très peu la réalité. 
Les idées, elles, s'apprivoisaient cependant à la pensée économique. Le principal mérite d'É. Montpetit fut de contribuer à changer un esprit qui rejetait à priori l'économique.

Le principal artisan du changement - changement dans l'ordre d'une plus grande structuration et d'une plus grande force intellectuelle - fut Lionel Groulx. Ce jeune clerc intelligent, un tantinet illuminé, dominera les années 20 et 30 de son dynamisme et de sa persévérance. Ne craignant pas de braver l'autorité - calmement bien sûr mais avec grande conviction il groupera autour de lui des hommes intelligents qu'il mettra au service d'une pensée nationale. Ayant compris très tôt qu'il fallait sortir du gouffre intellectuel collectif dans lequel s'enfonçait la société québécoise, il part à l'attaque: "Le vent est à l'action, aux méthodes énergiques ${ }^{8}$. "C'est à son dévouement sans bornes, à sa détermination et à son intelligence que nous devons les premières études d'importance sur la société québécoise. Sa volonté «aristocratique"s'attaquera surtout à trouver et à former une élite capable de comprendre un peuple, de reconnaître son destin et de l'y mener sûrement. Parce que "toute action libératrice procède des penseurs à la foule ${ }^{8}$ ", Lionel Groulx cherchera "les penseurs" et les aidera à trouver le droit chemin. Les voies qu'il tracera nous sont quelque peu familières çependant: "ll faudra bien que nous soyons de chez nous et de notre passé, si nous voulons continuer quelque chose ${ }^{9}$. "Cette dimension autocentrique nous surprend un peu chez un penseur dont l'élan nous annonçait un programme aux dimensions plus vastes. Mais le directeur du mouvement de l'Action française reste attaché à certains postulats qui ne se sont pas renouvelés depuis Casgrain: «Notre littérature de demain, catholique et française, se fera aussi bravement régionaliste ${ }^{10}$."

Avec Groulx, notre vocation surnaturelle» existe toujours. Notre statut d'État “ catholique et français ${ }^{11}$ " n'est pas qu'un vain titre de gloire; il comporte des devoirs et des règles auxquels notre destin nous lie. Et si la nation canadienne-française veut se montrer digne de ses origines, elle doit accepter de soumettre son destin à certaines conditions. La première de ces conditions est qu'aux niveaux individuel et collectif, tous s'efforcent de conserver cette «âme particulière» dont ils sont les dépositaires. La particularité de cette âme est que, pour conserver toute sa pureté, toute son authenticité virginale, elle doit se garder aussi des contacts corrupteurs qui pourraient l'entraîner dans un abâtardissement de mauvais aloi : "échapper à tout contact, à toute compression où cette âme pourrait mourir ou subir d'irréparables transformations ${ }^{12}$. Une pierre à la fois aussi précieuse et aussi fragile doit être conservée dans un écrin protecteur que seuls pourront ouvrir ceux qui en connaissent la valeur. Aussi faut-il donner au peuple une forteresse bien gardée. Ce sera un État indépendant où la nationalité, “maîtresse absolue de sa vie ${ }^{13}$ ", pourra se conserver dans toute sa splendeur. II ne s'agira que de régler savamment le thermostat d'une serre bien chauffée afin que le peuple puisse entendre la voix de ses maîtres du présent et du passé qui lui “inspirent de durer selon la loi de leur sang ${ }^{14}$ ". Lionel Groulx n'aime pas les demi-mesures. Pour lui, il faut être tout entier 
ou ne pas être. S'adressant à un groupe de bourgeois, leur parlant du rôle privilégié qu'ils ont à remplir pour la conservation (nous sommes près des musées) de l'âme québécoise, il aura ce mot terrible et juste:

Canadiens français, si nous ne pouvons l'être que d'une façon qui équivaut à ne pas l'être, qu'attend-on, en haut lieu, qu'attendent nos chefs pour nous le dire et pour nous commander de disparaître ${ }^{15}$.

Mais ce ne sont là que fleurs de rhétorique: l'auteur de la Naissance d'une race ne veut pas en voir la mort. La vie qu'il lui souhaite, avec toutes ses exigences de pureté, ne peut se garder cependant que sous un régime un peu spécial. Si "nous avons besoin d'une grande espérance qui traverse notre ciel et soulève enfin nos volontés ${ }^{16}$, il faut que tout un peuple se jette à genoux et implore les dieux à l'exemple de cette jeunesse à laquelle s'adresse l'instigateur de l'Action nationale:

Et vous, jeunesse, qui tant de fois nous avez déçus, mais qui toujours avez ranimé nos espoirs, faites que, par tous vos labeurs et par toutes vos prières, nous arrive ce qui, pour tout peuple trop affaissé est une indispensable condition de ressaisie, faites que nous arrive ce qui est arrivé au Portugal, à l'Espagne, à l'Irlande, à la Pologne, à I'Italie, même à la Turquie: un chef, un entraîneur, un excitateur d'enthousiasme et de volonté, qui soit aussi un calme ordonnateur d'énergies, un homme qui sache par quelle politique organique, persévérante, l'on sauve un pays; et alors, par vous, jeunesse, c'en sera fini de porter nos âmes en berne; nous les hisserons à hauteur d'homme, à la hauteur des grands Français nos aïeux ${ }^{17}$.

\section{DE JEAN-CHARLES HARVEY A ANDRÉ LAURENDEAU: ENTRE LE FASCISME ET LA RÉACTION}

Les appels à l'enthousiasme et à l'embrigadement lancés par l'Action française (nationale) ne rencontreront pas uniquement des oreilles de sourds. Un climat se crée d'où sortent des hommes et des idées, des mouvements même qui moduleront à leur façon les mots d'ordre lancés par Lionel Groulx. Vers les années 1930, les penseurs s'efforcent de laisser tomber l'idée de repos et veulent tendre les énergies vers une diversification et une universalisation de l'activité intellectuelle québécoise. L'Ordre d'Olivar Asselin est sur ce point l'organe le plus révélateur. Batailleur désintéressé, à la pensée guidée par un idéal jamais rabaissé, Olivar Asselin mènera une campagne d'approfondissement et de qualité sur tous les fronts:

Pour L'Ordre la renaissance nationale ne consistera pas uniquement, ou surtout, dans l'intensification de la natalité, mais dans le développement des plus hautes virtualités du peuple: intellectuelles, morales, même physiques ${ }^{18}$.

Mais les idées ont encore quelque difficulté à pénétrer la société québécoise. Toutes les difficultés que rencontre Jean-Charles Harvey illustrent jusqu'à quel point la société était encore proche du XIXe siècle. La lutte de l'auteur des Demi-civilisés en est une, au début, d'authenticité du vécu. 
“Soyez personnels, soyez vous-mêmes ${ }^{19}$ ", demandera-t-il à la jeunesse à qui revient le soin de changer les valeurs traditionnelles pour de nouvelles, plus conformes aux exigences des temps et des hommes modernes: "Donnez à la société à laquelle vous appartenez les éléments nouveaux que la vie a fait éclore dans votre sein ${ }^{20}$. "Que l'action dans laquelle se lance la jeunesse ne quitte pas des yeux "la vision de cette vierge lumineuse qu'on appelle l'idéal ${ }^{21}$ " afin que les «intelligences d'élite ${ }^{22}$ " puissent poursuivre noblement et sans défaillance cette longue marche collective qui chez Harvey, comme chez Groulx et Montpetit, comme dans toute la pensée québécoise doit conduire à «une incontestable supériorité». Pendant un certain temps, défiant tout compromis, J.-C. Harvey poursuivra malgré vents et marées, sa recherche. Quoi qu'il devienne lui-même, il passera cependant la main à toute une équipe qui pendant quelque temps sèmera la terreur dans les foyers traditionnels de la pensée. Un mélange de l'emballement de Groulx, des excès de J.-C. Harvey et de la rigueur d'O. Asselin allait se retrouver dans les pages rapides de Vivre sous la plume du rédacteur en chef: Jean-Louis Gagnon.

"À nous jeunesse", s'écriera-t-il dans le premier Cahier noir de la revue. Par cette exclamation, il veut rejoindre, près de lui, tout ce qui est «vivant» et capable d'affronter un avenir avec l'énergie nécessaire à la construction. Reconnaissant que "Asselin, Bourassa, Groulx, LaVergne sont des maîtres ${ }^{23}$ ", la direction de la revue nous propose son programme:

Vivre, c'est évoluer, c'est à dire, se défaire de tout esprit de conformisme, de toute idée en série, de tout préjugé. Voilà pourquoi vivre, c'est être jeune. C'est la révolte des générations qui montent et veulent inventer un état de vie nouveau, propre à elles, qui soit leur...24.

La jeunesse de 1930 semble en effet étouffer dans un carcan qui la limite et la contraint presque à la bêtise. Vivre - dans un mouvement d'humeur juvénile - veut sortir de l'ornière de silence que l'autoritarisme indigène recommande. Les jeunes «révolutionnaires»veulent “exprimer publiquement ce que pense à couvert une bonne partie de la jeunesse 25 ". Et ce que la jeunesse pense, c'est que l'orthodoxie a déjà assez duré et qu'il est temps que quelque chose change et que la pluralité des idées s'installe:

Nous ne pouvons continuer d'accepter comme de vieilles anglaises tartuffiées jusqu'aux moelles, les discours menteurs des métèques et des nôtres qui nous félicitent d'être resté un peuple profondément catholique ${ }^{26}$.

L'unité de pensée existe peut-être chez Vivre et ses rédacteurs, Pierre Chalout, Gérard Dagenais et autres, mais elle diffère de celle qui règne plus ou moins dans la société dans laquelle ils vivent et dont les piètres caractéristiques ne semblent guère leur plaire:

Rendu au grand $\times[$ sic] de sa destinée, le Pays de Québec sent que le temps est venu de mettre un point final à cette idiotie qui dure depuis trois siècles: exister. La quêteucratie canayenne étant devenue, en effet, une sacrée tradition sacrée en passe de devenir un protocole ${ }^{27}$. 
Jean-Louis Gagnon et son équipe veulent du neuf. Révolutionnaires, ils semblent bien l'être. Mais il semble aussi que toute la société veuille respirer un air nouveau. Dans sa lettre d'accueil, Lionel Groulx se dit heureux de la naissance de la revue: "Enfin, Dieu soit loué! Voici surgir de tout côté du neuf, du vrai neuf ${ }^{28}$." J.-M.-Rodrigue Villeneuve lui-même, évêque de Québec, tenant de l'orthodoxie ex officio, s'emballera pour ce nouveau vent qui gonfle les voiles de la génération montante:

Notre jeunesse en a fini de son pieux conformisme. Elle veut la révolution des idées au moins... avant celle des états sociaux ${ }^{29}$.

La réforme, le renouvellement des idées sinon la révolution étaient dans l'air. Tous étaient fatigués de stagner dans les mêmes idées abstraites. Tous désiraient aussi un changement, moins dans les idées que dans les faits. Le fascisme annoncé par Lionel Groulx progressait, s'affirmait et devenait monnaie courante. Les jeunes rédacteurs de Vivre diront:

$\grave{A}$ vingt ans ce n'est pas l'âge de légiférer doctement, mais bien de faire un effort pour réaliser ce qu'il y a de juste et de vrai dans les principes émis par les doctrinaires ${ }^{30}$ !

Ils se glorifieront même d'être les premiers à approuver "la dictature hitlérienne ${ }^{31}$ ", croyant si grande la valeur de ce régime que le reconnaître devenait un honneur. II se trouve même un André Laurendeau pour vanter le régime mussolinien dans l'Action nationale des mêmes années. En 1942 d'ailleurs, Rex Desmarchais, l'un des principaux rédacteurs de Vivre, n'écrira-t-il pas avec la Chesnaie l'un des plus authentiques manifestes fascistes? Et ce, sous le régime du peu démocratique Maurice Duplessis. La nostalgie du “maître» restera longtemps au cœur des disciples, de droite et de gauche, de Lionel Groulx.

Parallèlement à ces courants d'idées, se développe tout un mouvement dont l'importance semble avoir été très grande pour les contemporains si l'on en juge par leur témoignage. Le Jeune-Canada, dont tous les membres sortent ou reçoivent un excellent accueil de la part de l'Action nationale, est un regroupement de forces nationalistes - de type traditionnel - de la jeunesse québécoise, dont le zèle et l'action sont tout entiers inspirés par la doctrine de Lionel Groulx: quelque chose comme sa phalange laïque. Pris encore dans des considérations d'ordre linguistique de style biculturel ce mouvement ne semble pas dépasser les réclames des premiers numéros de l'Action française pour les calendriers bilingues: des calendriers, on est passé à l'argent et aux chèques. Tout cela se fait avec une volonté tenace de faire reconnaître par les maîtres anglo-saxons l'existence du fait culturel francophone en prenant comme thèse de départ qu'il faut «se souvenir que nous ne serons maîtres chez nous que si nous devenons dignes de l'être ${ }^{32}$; ; cela se poursuit par une vigilance de tous les moments dans tous les domaines, avec comme but premier «d'abord la mission apostolique du Canada français à laquelle (ils) croient beaucoup. Ensuite de propager la civilisation française ${ }^{33}$ ". Avec le JeuneCanada cependant, nous retournons quelque peu en arrière et nous ou- 
blions Bouchette, Parent et Montpetit pour nous attarder à une défense “réactionnaire" de la civilisation québécoise: "La société industrielle et capitaliste plonge ses victimes dans l'impossibilité d'accéder aux valeurs spirituelles ${ }^{34}$." Comme A.-B. Routhier, entre le progrès et la “conservation" nationale, le jeune A. Laurendeau n'hésite pas, il sacrifie le progrès, car ades ouvriers moins pauvres ne seront pas pour autant des ouvriers nationalistes ${ }^{35}$ ». Cette nouvelle aliénation au réel, vis-à-vis de laquelle Laurendeau prendra ses distances, nous amène directement à la philosophie de la Relève. Le critique économique de la Relève, Roger Duhamel, avait jugé ainsi le mouvement Jeune-Canada:

L'historien des idées au Canada français (s'il existe jamais!), constatera qu'une bonne part du réveil auquel nous assistons est due à cette cellule d'élite connue sous le nom de Jeune-Canada ${ }^{36}$.

En même temps que l'importance du courant d'idées, cette louange nous montre le lien idéologique entre le mouvement et la revue qui porte ce jugement.

\section{AUTOUR DE LA RELÈVE: INTELLECTUALISME ET RÉGRESSION}

Les esprits qui se groupént autour de la Relève sont tous entraînés dans un élan spirituel dont la principale caractéristique est la mise en valeur de l'individu dans un mépris hautain de la matière et du monde moderne. Fatigués de l'embrigadement national, tout comme le furent les artistes de l'École littéraire de Montréal, les jeunes Outremontois de la revue vont militer pour l'édification "d'un art; d'une littérature et d'une pensée dont l'absence commence à leur peser ${ }^{37}$ ". Dédaigneux de tout ce fatras régionaliste que constituent les scribouillages de leurs prédécesseurs canadiens-français, tournés vers une pensée européenne française qui correspond mieux à leur exigence intellectuelle, à l'écoute de philosophes sérieux, ils vont militer en faveur d'un personnalisme qui se souviendrait d'un Thomas d'Aquin modernisé. Leurs principales préoccupations sont d'ordre religieux dans l'engagement sérieux de tout leur être:

Notre catholicisme ne s'oppose pas à un art personnel, il le dépasse comme il dépasse une politique nationale: mais il s'y appuie comme sur la personne humaine ${ }^{38}$.

Dans les structures architectoniques de leurs valeurs, les jeunes intellectuels placent, à la toute pointe, leur engagement chrétien, ce qui les libère de préoccupations plus immédiates, plus terre à terre comme le politique et le social. En vivant le christianisme, ils ont la conviction de dépasser les préoccupations matérialistes et de redonner au monde sa véritable direction, son authentique raison d'être:

LA RELÈVE entend jouer un rôle social en rendant pour sa part dans le monde la primauté au spirituel ${ }^{39}$.

Forts de ce credo et libérés des préoccupations sociales et politiques, les jeunes collégiens se donneront tout entiers au développement et au 
culte de leur personne. L'individu a comme rôle social non pas de se lancer dans les grandes réformes, mais de développer son individualité car c'est son épanouissement qui apporte le plus à son milieu. A la Relève, le social n'est que l'addition d'individualités autonomes:

Je crois que je sauverai ma patrie en me sauvant moi-même ${ }^{40}$.

Cet individualisme “social» mènera la rédaction de la Relève à négliger le national, et ce, toujours pour la même raison: ses préoccupations sont loin de ce stade vulgaire et les valeurs qu'elle cherche contiennent et drainent avec elles, en les transcendant, les valeurs inférieures:

Les problèmes de la personnalité et de la culture nous ont paru préalables à l'action nationale ${ }^{41}$.

La personnalité et la culture étant prioritaires, tous les autres problèmes sont réglés, y compris le social et le national.

Avec des principes dont la particularité première est de vouloir s'embarrasser le moins possible des contingences matérielles, il n'est pas étonnant que le courant d'idées s'engage de plus en plus dans les ornières irréversibles d'un retour au passé, d'un retour à l'âge d'or où l'homme libéré des entraves de la réalité se retrouve tout entier dans l'unique quête du spirituel. Cet âge d'or sera bien entendu ce Moyen-Âge mythique que nous livre une histoire édulcorée ou un rêve désincarné. L'histoire de l'humanité au cours des cinq derniers siècles enseigne à la Relève que le rềve de l'homme de pouvoir se libérer complètement de toute dépendance envers Dieu conduit d'abord au néant d'un humanisme «qui vide l'homme au lieu de l'élever $42 \%$. En perdant l'unité multidimensionnelle du Moyen-Âge, l'homme se perd dans l'individualisme nietzschéen ou dans le socialisme marxiste, perdant dans les deux cas l'unité naturelle de la personne. Le socialisme n'étant qu'une réduction de l'homme en une “catégorie économique », il ne saurait représenter ni un progrès pour l'humanité, ni une voie pour l'avenir. Si l'humanisme consomme son échec dans le socialisme, il faut au plus tôt opérer une marche arrière et revenir à un stade antérieur où l'homme, retrouvant son unité dans une soumission intelligente à Dieu, va enfin pouvoir retrouver la paix et le bonheur que ses velléités d'indépendance lui ont fait perdre. Des pestes, des famines, des guerres du Moyen-Âge, la Relève ne dit mot. De retour à la monarchie paternaliste, I'homme, ayant retrouvé la simplicité et l'harmonie des temps révolus, pourra à nouveau vivre dans "une société plus laborieuse, à base de travail qualitatif auquel elle accordera son sens religieux ${ }^{43_{\text {}}}$.

Cette régression à un stade antérieur où le bonheur et la vie ne deviennent possibles que dans le rejet du présent va conduire une certaine forme de la pensée québécoise à cette maladie intellectuelle et sociale que fut la pensée de Saint-Denys Garneau:

Il faut donc atteindre à cette possession de soi par soi, progressivement et dans le temps ${ }^{44}$.

Cette nouvelle androgynie platonicienne qui verse dans le nombrilisme allait amener toute une génération d'écrivains à consacrer leur vie et leurs 
écrits à se défendre d'un engagement collectif dont ils n'avaient que faire et de préoccupations matérielles qu'ils méprisaient. "Le splendide isolement, c'est la mort ", écrira Robert Élie dans une préface à l'Homme d'ici d'Ernest Gagnon. On ne peut s'empêcher en lisant cette remarque de penser au correspondant et ami de l'auteur, Saint-Denys Garneau, et à sa pensée dont l'aboutissement ressemble à sa vie. Le rejet du national, qui n'est pas un mal en soi, devient chez l'auteur de Regards et jeux dans l'espace (titre fort éloquent) une modulation du rejet de soi qui le hante, lui et toute cette génération:

Faire des Canadiens français est une notion qui à peut-être cours mais qui n'a aucun sens. Elle est même à contre sens et contre nature. On peut prendre conscience de soi pour se donner, se parfaire: mais non pas pour se parfaire soi, mais bien pour se parfaire homme. D'ailleurs, on devient soi non pas tant en se cherchant qu'en agissant. Tout mouvement vers soi est stérile ${ }^{45}$.

Il est d'ailleurs à propos de la Relève une observation inquiétante à faire. Ces jeunes, les mieux éduqués et les plus brillants de leur génération et des générations précédentes, seront absolument bloqués, et ce pendant longtemps, face à ces étrangers qu'ils admirent - français surtout et parmi les plus célèbres - et à qui ils ouvrent toutes grandes les pages de leur revue. Lorsque la guerre se déclenche et que les intellectuels européens s'exilent en partie au Canada et aux États-Unis, il est remarquable de constater que servilement les rédacteurs de la Relève se retirent et laissent timidement leurs feuilles se noircir de l'écriture étrangère comme si honteux d'être ce qu'ils sont, ils craignaient de diminuer la valeur des Maîtres étrangers en plaçant leurs «pauvres cogitations» près des “Oracles" infiniment “supérieurs". Il ne retrouveront leur éloquence - et la coïncidence n'a de surprise pour personne - que lorsque la guerre finie, les “Maîtres" d'hier auront retrouvé d'autres revues européennes plus prestigieuses pour imprimer leur pensée. Alors les gens de la Relève recommenceront à écrire et à rédiger leur revue... délaissée par les «Maîtres».

\section{LE COOPÉRATISME}

Nous pouvons constater un lien facile entre les intellectuels de la Relève et ceux de Vivre: dans les deux clans, on se révoltait contre la pensée traditionnelle québécoise. Pour les rédacteurs de Vivre, ce rejet est une volonté de transformer cette pensée en un climat intellectuel plus viril, plus critique et plus sain. À la Relève, le rejet est plus catégorique: il est négation puis oubli de cette pensée, noyade enfin dans un intellectualisme spiritualiste et universel. Mais une constatation plus importante est à signaler ici: il s'agit dans les deux mouvements de querelles de chapelles. Ce sont des intellectuels qui se battent contre des intellectuels sur une question d'idées. Toutes ces contestations n'étaient que révolte de palais... On voulait changer le climat intellectuel sans connaître ou 
reconnaître le vrai mal, celui de la pauvreté matérielle dans laquelle croupissait le peuple québécois. Mais sans doute s'agissait-il là encore d'une évolution nécessaire: il fallait peut-être avoir tenté une révolution purement idéologique pour comprendre que cette révolution ne pouvait s'accomplir qu'après la révolution matérielle: celle qui, donnant du pain au peuple, allait lui permettre de “penser». II aura fallu bien du temps et quelques échecs à ces scolastiques pour se souvenir du primo vivere du Philosophe. D'ailleurs la terrible faillite économique des années 30 allait les aider à se rappeler cette vérité triviale que des penseurs comme $\mathrm{E}$. Bouchette ou É. Parent avaient pourtant déjà signalée... mais avec quel succès!

Même si les écrits de Saint-Denys Garneau et de la Relève ne nous le laissent guère pressentir, les années 30 furent, pour la société québécoise comme pour toute la société nord-américaine, une difficile décennie. Plus particulièrement ici où le peuple nouvellement prolétarisé venait d'entrer dans l'ère industrielle par la porte de service. L'économie québécoise est une réelle faillite. Le gouvernement et l'élite peuvent bien encore parler de colonisation: cela montre plus leur incapacité à diriger que leur bonne volonté. Mais l'élite et les dirigeants sont devant un dilemme inextricable. D'une part la politique agriculturiste est un échec: les terres sont maigres, improductives et incapables de récompenser le colon de son dur labeur, insuffisantes à le nourrir; d'autre part, l'idéologie traditionnelle a toujours condamné, comme antinationale, l'industrialisation et son corollaire, l'urbanisation. Mais quoi que dise, pense ou fasse l'élite, le processus de modernisation est engagé d'une façon irréversible. Entre un agriculturisme qui est une faillite et une urbanisation qu'elle condamne, la pensée traditionnelle québécoise mourra-t-elle d'indécision? Non, elle trouvera une solution qui règle non seulement le dilemme économique mais le problème philosophicopolitique qui en était né: la tentation du socialisme et du syndicalisme. Le corporatisme, ou le coopératisme, allait régler tous les problèmes en redonnant aux intellectuels une nouvelle tribune, à la société un nouveau rêve. Et dans les yeux de chaque lévite apparaissait le mirage d'une richesse qui s'était, jusqu'à maintenant et malgré les promesses de l'élite, tenue toujours trop loin:

La formule coopérative ne se présente-t-elle pas actuellement comme la plus apte à nous tirer des décombres d'un capitalisme vicié comme à nous préserver des pseudo réformes d'un socialisme révolutionnaire ${ }^{46}$.

Cette découverte comblera tout le monde: les penseurs et les hommes publics s'y précipitèrent comme des chacals sur l'unique charogne d'un désert... idéologique.

Fort de l'appui, de l'encouragement et de la propagande de l'Action nationale, le coopératisme se voulait d'abord une réponse saine aux malaises d'une société en voie de décomposition ou en voie de restructuration. Tous les maux dont souffre la société sont mis sur le dos du libéralisme économique qui sera perçu comme une véritable jungle déshumanisante où l'homme n'est qu'un loup pour l'homme et où la liberté n'en- 
gendre que l'exploitation en encourageant l'égoïsme et l'assouvissement des bas instincts de domination. Cet individualisme qu'engendre le libéralisme n'est bon qu'à tuer ce qu'il y a de plus grand dans l'homme, son sens de la charité et de l'entraide. Dans les faubourgs industrieux, on ne voit plus ces beaux gestes que l'on retrouvait à la campagne: les "corvées", les réunions où tous oublient leur intérêt particulier mesquin pour se donner dans la joie à une œuvre altruiste. La lutte au libéralisme ne doit cependant pas mener à un socialisme égalitaire qui conduirait nécessairement à l'abandon des édifiantes et louables traditions de notre bon peuple:

La socialisation des services médicaux, des hôpitaux, etc.: c'està-dire la mort de nos admirables institutions chrétiennes de bienfaisance, l'affaiblissement de la charité privée et du dévouement... ${ }^{47}$.

Dans la vie, dirait-on, it y a des choses bonnes et des choses mauvaises. Le socialisme est mauvais. La libre entreprise et le monopole des professions libérales et cléricales sont bons: ne gardent-ils pas le mérite de respecter la parole de l'Évangile qui nous enseigne qu'il y aura toujours des pauvres parmi nous? Et sur qui s'exerceraient la charité des riches et le dévouement des bonnes sœurs si les pauvres disparaissaient? Contre «l'individualisme meurtrier qui a engendré le libéralisme économique ${ }^{48}$ 》 bien sûr! Mais il y a des limites à cette opposition! II y a des choses qui ne se font pas! Telle semble être la morale un peu simpliste, sur certains points, de Georges-Henri Lévesque.

Le fait est qu'au fond, l'on combat moins le libéralisme que le danger montant de l'influence du socialisme communiste et du syndicalisme international «athée». Parti en chasse contre le matérialisme - en cela les théories de Georges-Henri Lévesque se rattachent à la pensée traditionnelle - les coopératistes réfutent à grands coups de goupillon les préoccupations bassement matérielles des systèmes socialistes, socialisants et socialisateurs. Leur phobie du matérialisme qui fait condamner le marxisme sous toutes ses formes, les conduit en même temps - on ne sait selon quel sens logique - à craindre par-dessus tout dans les réformes socialistes "la perte de la propriété privée» comme si la «propriété" était un symbole de l'anti-matérialisme. La logique qui les mène à une telle conclusion ressemble fort à celle qui leur fait imaginer la nécessité des pauvres et des défavorisés pour que le sens “charitable» des riches puisse s'exercer. À la peur du matérialisme, à celle de l'étatisation se joint la hantise de l'étatisme dans lequel toutes les libertés individuelles sont embrigadées non pas pour l'accomplissement de leur salut - ce qui est bien - mais pour le service d'un État qui, comme en Russie, oublie le bien particulier pour s'adonner à l'édification d'une société qui centralise et oriente toutes les énergies pour le bien de l'État - ce qui est mal. Toutes ces craintes encore plus que les malaises sociaux allaient précipiter la pensée québécoise dans le coopératisme.

Quelles que soient les raisons psychologiques, sociales et religieuses qui amènent le coopératisme, l'intention était louable et le moyen 
gardait une certaine efficacité. Devant la pauvreté quasi universelle du peuple québécois, les collaborateurs de Georges-Henri Lévesque, plutôt que de parler de résignation, de récompense et de paradis, cherchèrent un remède. La pauvreté avait un grand désavantage, c'est qu'elle éloignait l'individu de son centre de contrôle et de préservation: la paroisse. Elle le précipitait à la ville où il ne devenait pas plus riche mais où il perdait les secours "moraux" du "père" de la paroisse et se laissait aller aux influences étrangères néfastes. La meilleure façon de contrebalancer cet exode, qui de fait durait depuis cent ans, c'était de donner au peuple une prospérité qu'il pouvait acquérir dans son milieu naturel. L'agriculturisme ne faisant plus l'affaire, l'école économique va s'efforcer, par des regroupements, de rendre au peuple le profit que centralisent quelques capitalistes détenteurs de certains monopoles laitiers, forestiers ou industriels. Cette redistribution de richesses, en même temps qu'elle enseigne la collaboration, "la charité" comme dit le Père Lévesque, permettrait au peuple de recouvrer une certaine décence matérielle en l'éloignant de la tentation de la ville et de l'exil.

Mais qu'est-ce que le coopératisme sinon un socialisme, honteux de lui-même, qui vient de l'orthodoxie? On découvre qu'il n'y a qu'un moyen de se sauver de la domination du libéralisme économique et c'est de s'unir. "Unissons-nous», disent les coopératistes mais évitons l'embrigadement à la russe et, tout en joignant nos efforts à la fois pour nous libérer et progresser, respectons les «individualités» et évitons de nous subordonner à un ordre qui néglige “les véritables valeurs humaines», les valeurs spirituelles. Il faut "planifier", mais ne tombons pas dans le piège de l'étatisme qui, subordonnant toute l'activité humaine à la volonté de l'État laïque et matériel, néglige l'âme. Libérons l'économie de la mainmise capitaliste, mais n'oublions pas que certaines valeurs, certaines traditions, certaines corporations établies doivent être respectées... et que Dieu de toute façon n'a pas créé les hommes égaux. Seule forme de socialisme acceptable dans une société encore trop proche d'un MoyenÂge qui se prolonge, le coopératisme avait l'avantage de laisser se réaliser certaines réformes nécessaires, dans un pays où l'économie et l'idéologie prêchées depuis plus de cent ans s'étaient avérées incapables de répondre aux besoins de la société. Mais le coopératisme, au point de vue purement idéologique, est surtout intéressant pour le fait qu'il répondait aux exigences de la triple appartenance ou dépendance de la pensée canadiennefrançaise. En s'appuyant sur l'encyclique Quadragesimo anno, on montrait le respect que l'on gardait vis-à-vis de l'autorité romaine. En s'inspirant de la pensée catholique française, on satisfaisait au paternalisme culturel français. En se basant sur les études menées par «Les Semaines sociales du Canada", on remplissait les exigences des velléités d'originalité régionaliste. Le coopératisme arrivait à point et se mariait intimement aux exigences et aux particularités de la pensée et de la réalité québécoise, à un point tel qu'il fut déclaré d'intérêt national: 
Un front commun corporatiste, c'est une nécessité nationale pour nous, Canadiens français ${ }^{49}$.

décrétait l'éditorial de l'Action nationale de décembre 1938.

\section{LA VIEILLE GARDE À L'AFFÛT: C.-H. GRIGNON, F.-A. SAVARD}

Le coopératisme, par-delà sa crainte et son incapacité d'aller au fond des choses et des mots, demeurait un effort positif dans le domaine de la réforme socio-économique. Il y avait au moins, d'une façon discrète et implicite, une constatation sinon une acceptation de l'échec de la politique et de l'idéologie traditionnelles. C'était un effort positif qui tentait d'orienter, gauchement, le Québec dans un courant «contemporain»: c'était une certaine forme de socialisme. Parallèlement à cet effort, des voix isolées - mais qui trouveront des oreilles attentives - profitant des malheurs du temps, s'élèveront pour chanter le péan d'un nouveau retour en arrière. Attribuant tous les maux du pays au fait que l'on ait trahi le passé, ces «bardes folkloristes "s'acharneront à jouer les profiteurs de deuils et les conservateurs de musée. Claude-Henri Grignon, (Valdombre) fort de son prestige populaire et de sa renommée de pamphlétaire agressif, sera le représentant le plus lourd et le plus buté de cette nouvelle vague réactionnaire. Toute l'industrialisation, toute l'ouverture au monde elle n'est encore que porte entrouverte - tous les changements ne seront pour lui qu'une façon de trahir les ancêtres, de renier les siens. Vivant encore du passé, tant pécuniairement, avec son roman feuilleton, que moralement, il ne voit l'avenir québécois que comme une vaste fixation, une continuelle contemplation d'un soi archaïque. Le changement, l'évolution pour lui, c'est une trahison, une perte de l'authenticité:

II ne faut pas que le vieux Québec change. C'est à souhaiter plutôt qu'il se fasse plus vieux que jamais, qu'il retourne à son passé comme à une source inépuisable de richesse économique et d'inspiration poétique ${ }^{50}$.

Du passé lui-même, l'auteur d'Un homme et son péché ne retient que ce qu'il a de moins rentable, de plus mesquin et de plus stérile: le "sol" québécois, dont les plus belles récoltes sont les roches qui, comme des dolmens effrités, trônent au milieu des champs maigres et poussiéreux. Malgré les faits, Valdombre s'obstine à trouver, dans le retour à la terre, le salut à tous les maux de la société, qu'ils soient sociaux, économiques ou moraux. Loin de la contamination du monde moderne, tout près de ses sources, réchauffé par les mânes de ses pieux et valeureux pères, le colon canadien-français, restant lui-même, sauvera son être d'une évolution qui serait pour lui une mort certaine:

Notre survivance reste intimement liée au sol. Le mot "sol" (trois lettres) contient tout le passé, toutes nos traditions, nos mœurs, notre foi et notre langue. Retranchez le sol de notre vie sociale, économique, politique et il n'est point de culture canadienne-française ${ }^{51}$. 
Cette culture canadienne-française a besoin d'un véhicule linguistique qui doit lui être particulier. Tant et aussi longtemps qu'on s'efforcera de faire vivre notre caractère singulier dans une langue qui n'est point nôtre, la langue de Molière, nous nous égarerons. Une culture propre a son instrument propre: une langue «différente". Et c'est toute l'apologie de la langue du terroir: "Nous avons besoin d'une langue bien à nous, une langue du terroir 52. "Un rétrécissement des valeurs et de l'être à ce qu'il y a de plus petit et de plus mesquin: la conservation de soi par ses défauts les plus "pittoresques" et un rejet de l' "étrange» comme d'une infection. Toutes choses, que d'une façon symbolique, nous retrouvons chez le célèbre Pierre-Côme Provençal du Survenant, dont la condamnation de l'étranger ressemble fort à un rejet d'un monde en pleine mutation:

Sûrement Didace avait eu une heure malheureuse quand il avait accepté le Survenant, ce chef-d'œuvreux, dans la maison. Rien de bon $n$ 'en avait résulté pour la paroisse. Une si belle paroisse que les anciens avaient bâtie avec tant de cceur. Si l'on veut la garder ainsi entre soi, il ne faut pas laisser l'étranger y pénétrer et en faire une risée. Autrement on la voue à sa perte ${ }^{53}$.

Si en 1941, Claude-Henri Grignon peut tenir ces propos sans se couvrir de ridicule, c'est que tout un groupe de personnes de son acabit encourage et diffuse la volonté d'un retour en arrière qu'ils prêchent comme une condition sine qua non de survie. À peine deux ans plus tard, en 1943, Félix-Antoine Savard ne craindra pas lui non plus de faire l'éloge de cette Église qui participa activement à la campagne de colonisation de l'Abitibi. L'urbanisation et l'industrialisation avaient suivi leur cours normal malgré les tirades oratoires des "agriculturistes" de toute robe. Mais lorsque la crise était survenue, toutes ces voix dans le désert avaient trouvé leur vengeance: la faillite économique des années 30 était pour eux l'occasion de crier sur tous les toits: “On vous l'avait bien dit. "Qui plus est, pour eux, cette faillite était la preuve même que les tenants du "retour à la terre" avaient raison. Selon une habitude illogique de la logique scolastique, prouver que l'adversaire a tort, c'est se prouver à soi et aux autres que l'on a raison:

On se résolut enfin à écouter l'Église, à entendre, dans la disette, des économistes qu'on avait méprisés dans l'abondance. Et la croisade du retour à la terre fut décrétée ${ }^{54}$.

A la satisfaction de posséder la vérité et au plaisir d'être écouté dans ces temps tristes, Félix-Antoine Savard allait ajouter une note presque inimaginable si l'on se resitue à l'époque terrible des années d'avant-guerre. Au moment où toute la pensée québécoise a la preuve de son échec, alors que la société est une faillite monumentale, que la morale elle-même est d'un primitivisme déconcertant, sauf pour quelques élus dédaigneux, l'auteur de Menaud resservira cette vieille soupe du mythe de la supériorité:

Si nous perdîmes un empire, l'héritage nous fut confié de la civilisation la plus humaine et la plus proche de l'Evangile; la plus pacifique et la plus respectueuse du bien d'autrui ${ }^{55}$. 
De ce riche héritage, il ne restait plus grand-chose, si héritage il y eut jamais, qu'un peuple pauvre et misérable à tous les points de vue.

La période des années 40 allait d'ailleurs être dominée par un homme dont l'absence de dimension intellectuelle allait être le premier titre de gloire: Maurice Duplessis. Évitant de penser et préservant le plus possible le peuple de toute activité intelligente, cet homme petit allait faire régner la bêtise pendant plus de vingt ans. Cette responsabilité, il la partagera d'ailleurs avec son parti qui devait camoufler son impuissance politique sous les discours patriotiques enflammés et le bitume routier. Portait aussi sa part de responsabilité toute la pensée traditionnelle qui, pour un chiffon fleurdelisé, quelques pieds-de-nez au gouvernement fédéral et des subventions ridicules, allait se laisser acheter un silence complice. II ne faudrait pas oublier non plus dans le partage du butin ce peuple qui se laissait berner si facilement et dont la bêtise et l'ignorance - quelle qu'en soit la cause - furent les grands responsables de cette longue période de grande noirceur. Ce parti, ces intellectuels, ce peuple, tous écoutaient, bouche bée ce premier ministre - qui les représentait fort bien - chantant ces mots inimaginables:

La province de Québec n'a pas de supérieure au monde! Elle est honnête, consciencieuse, respectueuse des lois et de l'autorité établie. Et sa réputation est bien au-dessus de certaines déclarations dont il ne faut pas tenir compte, mais qu'il faut souligner discrètement... Quand j'ai été invité à l'inauguration du Pavillon de la faculté des sciences à l'université de Sherbrooke, j'en ai ressenti un vif plaisir. Bien que je sois célibataire, j'ai l'impression d'être venu voir un de mes enfants, brillant et prometteur et dont la croissance ajoutera au lustre de la province de Québec... ${ }^{56}$.

Toute cette époque en fut une des plus pitoyables au niveau de la pensée officielle. C'était le moment où les communistes faisaient sauter les ponts, où les Anglais d'Ottawa étaient la cause des déficiences économiques et où le bon peuple innocent s'amusait des calembours douteux d'un homme de piètre intelligence mais doué d'un sens opportuniste inégalable. Cependant, des ingénieurs incompétents commettaient des erreurs impunies - sur les ponts et ailleurs - en faisant des millions, et des politiciens véreux faisaient de leur ignorance un art politique.

Pendant ce temps, les faits changent sous leurs yeux, et ils n'ont rien vu, et ils $n^{\prime}$ ont rien fait ${ }^{57}$.

écrira Paul Chamberland quelques années plus tard avec combien de justesse.

\section{LA MUTATION: UN MONDE S'ÉVEILLE AU MONDE}

Pendant que certains ratiocineurs se morfondaient à oublier le monde et l'histoire, d'autres cependant, plus sensibles, sentirent le monde trembler sous leurs pieds. Quelle que fut leur nostalgie d'un passé reposant, quelle que fut leur espérance de changement, ces hommes, con- 
scients à leur façon et à leur empan, comprirent qu'il fallait bon gré, mal gré, entrer dans le jeu. La guerre, bouleversant le monde, avait bouleversé aussi le giron calme du Québec. Tout annonçait l'avènement d'un monde nouveau où plus jamais ne reviendrait le confort des serres surchauffées. Définitivement exclue de sa quiétude passée, la société québécoise, malgré quelques efforts tenaces pour l'en empêcher, allait entrer dans un monde nouveau. Les premiers pressentiments des changements inévitables et nécessaires se firent après la guerre et autour des années 1950. Ce fut comme une révélation:

Ils ont compris soudain que leur petit monde bucolique craquait de toutes ses solives sous la pression formidable du temps qui chan$\mathrm{ge}^{58}$.

Les premiers signes avant-coureurs nous viennent de cet André Laurendeau qui avait déjà écrit: “Catholiques: c'est tout dire, tout expliquer ${ }^{59}$." II est vrai que cet homme parti de très loin allait être de toute l'école traditionnelle le plus perceptible au changement et le plus disponible à l'évolution. II sera celui qui dès 1948 prévoyait toutes les luttes idéologiques des années 50 et 60 . Faisant une autopsie et un bilan du nationalisme, il constate combien celui-ci est impuissant devant la montée du monde moderne. Au capitalisme, il oppose une vertu digne peut-être mais qui a fort peu d'écho chez une population assoiffée de bien-être et de plaisir. À un syndicalisme revendicateur, le nationalisme n'offre rien qu'un sermon sur la grandeur d'être Canadien français... Enfin, aux préoccupations sociales, le nationalisme a préféré l'exaltation un peu boursouflée de valeurs bourgeoises qui ne correspondent en rien aux volontés et aux désirs des ouvriers. Bien conscient de tous ces problèmes, le futur éditorialiste du Devoir, l'un des principaux adversaires de Maurice Le Noblet Duplessis allait prophétiser en juin 1948:

Le social et le national se présentent actuellement comme s'ils étaient deux ennemis, ou du moins comme si chacun réclamait toute la place $^{60}$.

Cet article de Laurendeau nous amène directement au Refus global des amis de Borduas. Cette analyse nous montre aussi que le "refus" était dans l'air et que le "poème» de Borduas pour l'avoir exprimé d'une façon marquante en des mots enflammés était plus l'expression d'un milieu et d'un état d'esprit qu'un point de départ brusque et original. Ce qui n'enlève rien au mérite du peintre: il gardera toujours le mérite de l'expression d'un tournant dans la pensée québécoise. Son manifeste s'arrêtera d'abord à condamner ce «destin durement fixé de la tradition». Le rejet d'un passé fait de négation, d'interdit et de passivité l'oblige à condamner la «tutelle» et l'autoritarisme dominant de l'élite et d'une classe aveugles: "Au diable le goupillon et la tuque»! leur hégémonie, celle du folklore et de la morale, ne nous a conduits qu'à une impasse. S'ils furent utiles jadis, minimement, ils ont depuis, par leur sclérose et leur étroitesse d'esprit, fait plus de tort que de bien, infiniment plus: "Mille fois ils extorquèrent ce qu'ils donnèrent jadis ${ }^{61}$. "Le Refus malgré son titre est d'une 
inspiration toute tournée vers le mouvement et la vie. Que les fixations pathologiques, que les tabous s'écroulent pour laisser place à un soleil régénérateur. Que "toutes les libertés possibles" s'animent et qu'elles soient disponibles même au prix fort des hautes luttes et de l'angoisse. C'est de ce "sauvage besoin de libération" que l'espoir collectif d'un monde meilleur naîtra. Mais bien que les valeurs changent, bien que l'ouverture au monde se fasse toute grande, la réalité sociale d'un peuple petit numériquement demeure: aussi voit-on revenir chez Borduas l'appel à l'union de toutes les forces disponibles qui nous fait nous ressouvenir d'un certain unanimisme. De même, l'appel à la responsabilité collective qui porte encore le relent du messianisme ne peut être évité : “Un magnifique devoir nous incombe ${ }^{62}$." Ce devoir est fait d'un dynamisme tourné vers le futur dont l'Utopie, pour être plus moderne, n'est pas sans quelque rapport avec celle de Henri-Raymond Casgrain.

Le mouvement en avant est désormais irréversible. Un clerc jésuite participera lui-même à l'expression de la liberté. Ernest Gagnon dans l'Homme d'ici, en 1963, lancera tout un projet visant à relever l'homme québécois de sa chute dans l'insignifiance. Son homme à lui aura comme base non plus «l'absolu» mais le "relatif». Une relativité qui place l'homme en une "genèse» dont l'élan se fait vers l'avenir, vers le neuf. Son rejet de la "soumission morbide" et la condamnation de la "passivité" lui font rencontrer la dynamique de Borduas qu'il baptise d'un déterminisme théologique. Celui-ci cependant est plus un appel au mouvement et à l'effort qu'une paisible contemplation de la vérité. La condamnation qu'il lance sur la société traditionnelle a le réalisme et la sévérité de Lord Durham: "Un groupement d'excellents seconds ternes et obséquieux ${ }^{63}$ ", dira-t-il condamnant toute la philosophie qui avait ravalé tout un peuple à l'impuissance. Dans un élan qui garde la crainte du matérialisme, il provoquera la quête du quotidien que l'on avait si souvent oublié dans le retour au passé ou dans le rêve de l'avenir, demandant aux intellectuels et aux éducateurs de donner vie au peuple:

Il faut lui apprendre que ses forces de croissance ne résideront ni dans la solidité de son dollar, ni dans le sous-sol de l'Ungava, ni même dans un passé mort. Ses richesses d'adulte sont dans le présent de sa vie quotidienne ${ }^{64}$.

Par-delà le paternalisme latent de cette recommandation, nous pouvons voir l'esprit qui présidera à l'effort de «rattrapage " de l'équipe de Cité libre. Comme Laurendeau, Borduas et Hertel, Ernest Gagnon représente bien la prise de conscience que tout doit changer chez un peuple qui ne peut ou ne veut plus se contenter de bonnes paroles ${ }^{65}$.

Georges Vincenthier Collège de Sherbrooke

1. Rappelons que l'Action française paraît jusqu'en 1928, et l'Action nationale, en 1933. 
2. Pierre Elliott Trudeau, la Grève de l'amiante, Montréal, Cité libre, 1956, p. 13.

3. E. Minville, "Ce que nous voulons", l'Action française, vol. Vl, octobre 1936, p. 95.

4. E. Montpetit, "Vers la supériorité ", l'Action française, vol. I, $n^{\circ} 1,1917$, p. 1 et 5.

5. É. Montpetit, "Six jours à Berkeley», Revue trimestrielle canadienne, mai 1918 , p. 6.

6. Ibid., p. 17.

7. Lionel Groulx, "Une action intellectuelle», I'Action française, vol. I, $\mathrm{n}^{\circ} 2$ 1917 , p. 33.

8. Ibid., p. 35.

9. Ibid., p. 37.

10. Ibid., p. 39.

11. Lionel Groulx, Notre avenir politique, Montréal, L'Action française, 1923, p. 21.

12. Ibid., p. 22. Lire en ce sens l'Appel de la race, Montréal, L'Action française, 1922.

13. Ibid.

14. Ibid., p. 23.

15. Lionel Groulx, l'Avenir de notre bourgeoisie, Montréal, Valiquette, 1939, p. 112.

16. Id., Notre avenir politique, p. 30 .

17. Lionel Groulx, l'Avenir de notre bourgeoisie, p. 112.

18. Olivar Asselin, "En guise de programme ", l'Ordre, première année, n 1, 10 mars 1934, p. 2.

19. Jean-Charles Harvey, Pages critiques, Québec, Le Soleil, 1926, p. 182.

20. Ibid., p. 183.

21. Ibid., p. 182.

22. Ibid., p. 184.

23. La Direction, Vivre, première série, $n^{\circ} 6$, janvier 1935, p. 4.

24. La Direction, Vivre, deuxième série, $n^{\circ} 2,22$ mars 1935.

25. La Direction, Vivre, première série, $n^{\circ} 6$, janvier 1935, p. 3.

26. La Direction, Vivre, première série, $n^{\circ} 3$, p. 3.

27. Jean-Louis Gagnon, Introduction à J.-C. Harvey, Jeunesse, Ed. du Quotidien, "Les Cahiers noirs", Québec, 1935.

28. Lionel Groulx, «Témoignage ", Vivre, première série, $n^{\circ} 3, p .18$.

29. J.-M.-R. Villeneuve, propos cité dans "Editorial», Vivre, première série, $n^{\circ} 5$, décembre 1934, p. 3.

30. La Direction, Vivre, première série, $n^{\circ} 5$, janvier $1935, p .3$.

31. Pierre Chalout, Vivre, première série, $n^{\circ} 5$, décembre $1934, p .31$.

32. "Manifeste des jeunes", l'Action nationale, vol. I, février 1933, p. 120.

33. André Laurendeau, "le Jeune-Canada", interview accordée à Vivre, deuxième série, $n^{\circ} 3$, avril 1935 .

34. Id., "Conclusions très provisoires", l'Action nationale, $n^{\circ} 31$, juin 1948, p. 415.

35. Ibid., p. 417.

36. Roger Duhamel, la Relève, vol. II, $n^{\circ} 5,1936$, p. 152.

37. La Direction, «Positions", la Relève, vol. I, n ${ }^{\circ} 2$, p. 4.

38. Ibid., p. 4.

39. Ibid., p. 5.

40. A. Laurendeau, la Releve, vol. II, $n^{\circ} 2,1935$, p. 35.

41. La Relève, texte signé par l'équipe, premier cahier, $4^{e}$ série, novembre-décembre 1938, p. 194.

42. La Relève, “Un nouveau moyen-âge», texte signé par l'Équipe, cahier. $n^{\circ} 8$, 1935 , p. 210.

43. Ibid., p. 214.

44. La Relève, texte signé par l'Équipe, l, cahier $n^{\circ} 7$, p. 154.

45. H. de Saint-Denys Garneau, Journal, Montréal, Beauchemin, 1962, p. 205.

46. Georges-Henri Lévesque, "la Nouvelle Chaire de coopération à I'Université Laval", l'Action nationale, vol. XI, novembre 1938, p. 217.

47. Georges-Henri Lévesque, “Socialisme canadien", l'Action nationale, vol. II, octobre 1933, p. 112 .

48. Ibid., p. 93.

49. "Éditorial", l'Action nationale, vol. XI, décembre 1938.

50. Valdombre, "D'une culture canadienne-française", l'Action nationale, $n^{\circ} 17$, juin 1941, p. 540.

51. Ibid., p. 538.

52. Ibid., p. 541.

52. Ibid., p. 541. 
53. Germaine Guévremont, Marie Didace, Montréal, Fides, «Nénuphar», 1965, p. 136.

54. Félix-Antoine Savard, l'Abatis, Montréal, Fides, 1943, p. 14.

55. Ibid., p. 31.

56. Paroles de Maurice Duplessis lors de l'inauguration de la faculté des Sciences de l'Université de Sherbrooke en 1957 et rapportées par Cité libre, n 19, janvier 1958, p. 21.

57. Paul Chamberland, “De la damnation à la liberté », Parti pris, vol. I, nos 9-10-11, été 1964, p. 56.

58. Gérard Pelletier, Cité libre, vol. I, $n^{\circ} 2$, février 1951.

59. André Laurendeau, "Manifeste», le Semeur, nos 1-2, septembre-octobre $1934,31^{\ominus}$ année, p. 5.

60. André Laurendeau, "Conclusions très provisoires", p. 424.

61. Paul-Émile Borduas, Refus global, Saint-Hilaire, Mitramythe, 1948.

62. Ibid.

63. Ernest Gagnon, l'Homme d'ici, Montréal, HMH, 1963, p. 27.

64. Ibid., p. 25 .

65. Ce texte est extrait d'un essai à paraître bientôt chez $\mathrm{HMH}$, dans " les Cahiers du Québec ". 